\title{
Tissue Damage Markers after a Spinal Manipulation in Healthy Subjects: A Preliminary Report of a Randomized Controlled Trial
}

\author{
A. Achalandabaso, ${ }^{1}$ G. Plaza-Manzano, ${ }^{2}$ R. Lomas-Vega, ${ }^{3}$ A. Martínez-Amat, ${ }^{3}$ \\ M. V. Camacho, ${ }^{4}$ M. Gassó, ${ }^{4}$ F. Hita-Contreras, ${ }^{3}$ and F. Molina ${ }^{3}$ \\ ${ }^{1}$ Centro de Fisioterapia y Psicología Soluciona, 18002 Granada, Spain \\ ${ }^{2}$ Department of Medicine, Universidad Complutense de Madrid, 28040 Madrid, Spain \\ ${ }^{3}$ Department of Health Sciences, Universidad de Jaén, Paraje Las Lagunillas s/n, 23071 Jaén, Spain \\ ${ }^{4}$ Servicio de Análisis Clínicos, Hospital Médico-Quirúrgico del Complejo Hospitalario de Jaén, Servicio Andaluz de Salud, \\ 23007 Jaén, Spain \\ Correspondence should be addressed to F. Molina; fjmolina@ujaen.es
}

Received 25 September 2014; Accepted 10 December 2014; Published 25 December 2014

Academic Editor: Benoit Dugue

Copyright ( 2014 A. Achalandabaso et al. This is an open access article distributed under the Creative Commons Attribution License, which permits unrestricted use, distribution, and reproduction in any medium, provided the original work is properly cited.

Spinal manipulation (SM) is a manual therapy technique frequently applied to treat musculoskeletal disorders because of its analgesic effects. It is defined by a manual procedure involving a directed impulse to move a joint past its physiologic range of movement (ROM). In this sense, to exceed the physiologic ROM of a joint could trigger tissue damage, which might represent an adverse effect associated with spinal manipulation. The present work tries to explore the presence of tissue damage associated with SM through the damage markers analysis. Thirty healthy subjects recruited at the University of Jaén were submitted to a placebo SM (control group; $n=10$ ), a single lower cervical manipulation (cervical group; $n=10$ ), and a thoracic manipulation $(n=10)$. Before the intervention, blood samples were extracted and centrifuged to obtain plasma and serum. The procedure was repeated right after the intervention and two hours after the intervention. Tissue damage markers creatine phosphokinase (CPK), lactate dehydrogenase (LDH), C-reactive protein (CRP), troponin-I, myoglobin, neuron-specific enolase (NSE), and aldolase were determined in samples. Statistical analysis was performed through a $3 \times 3$ mixed-model ANOVA. Neither cervical manipulation nor thoracic manipulation did produce significant changes in the CPK, LDH, CRP, troponin-I, myoglobin, NSE, or aldolase blood levels. Our data suggest that the mechanical strain produced by SM seems to be innocuous to the joints and surrounding tissues in healthy subjects.

\section{Introduction}

Spinal manipulation (SM) is a common form of intervention used by a wide range of practitioners used to relieve pain and disability of the musculoskeletal system. A precise definition of SM is still under review. The SM is frequently defined as a manual procedure that involves a directed impulse to move a joint past its physiologic ROM without exceeding its anatomical limit $[1,2]$. Although its effectiveness has been demonstrated in some spinal syndromes such as $[3,4]$, several studies show concomitant organic complications with the application of cervical spinal manipulation (CSM) $[2,5,6]$.
Mild to moderate adverse effects occur in a large proportion of patients receiving spinal manipulation [7]. Although the majority of the adverse effects are transient and nonserious [7], severe adverse events such as cerebrovascular accidents and paraplegia have been associated with SM [8]. These injuries are typically described following an upper-back CSM. Although some authors have proposed that, in the presence of a marked degree of atherosclerosis, the mechanical stretching and compression effects of rotational manipulative techniques [9] may impose a further risk factor not only for vertebrobasilar insufficiency but also for lesions of the endothelium [10], recent studies suggest that SM may induce less 
arterial strain than the range of motion test when cervical rotation is examined [11].

Therefore, there are controversial data about the possibility of CSM inducing pathological mechanical stress that might in turn provoke vascular and neurological accidents [11-14]. Some evidence supports thoracic spinal manipulation (TSM) as an alternative to CSM to relieve pain and disability in the cervical spine [15]. There is no evidence of serious adverse events related to TSM. Given this situation, it is necessary to determine whether spinal manipulation is an innocuous technique.

Several proteins have been widely used in medicine as markers of tissue damage. These damage biomarkers are cell proteins or enzymes normally located inside specific cells. The detection of these proteins in serum and cerebrospinal fluid is a tell-tale of cell breakage produced by tissue damage. Proteins like creatine phosphokinase (CPK), lactate dehydrogenase (LDH), aldolase, myoglobin, and troponin-I [16] have been described as tissue damage markers in conditions such as strenuous exercise, brain injury, and heart damage [16-21], when altered levels of theses markers were detected in blood samples. CPK [22] and myoglobin [16] are the most widely used blood parameters for the detection of striated muscle injury although other parameters seem to be more sensitive to the difference between cardiac muscle and skeletal muscle damage. In this respect, the skeletal muscle troponin-I subunit may be an earlier and more suitable marker for skeletal muscle damage than CPK [23] and it is widely used as a muscle damage maker after strenuous exercise [24].

On the other hand, neuron-specific enolase (NSE) is also a glycolytic enzyme, which occurs in neurons and axons and is an appropriate marker for neuronal damage [25].

Finally, C-reactive protein (CRP) is a widely employed systemic marker of inflammation and tissue damage [26].

The aim of this preliminary study is to determine the possible noxious effects of spinal manipulation. To the extent of our knowledge, this is the first work that focused on the study of SM and mechanically induced tissue damage through the analysis of damage biomarkers in blood samples.

\section{Methods}

2.1. Design Overview. It is randomized repeated-measures controlled trial.

2.2. Setting and Participants. The study was approved by the Ethics Committee of the University of Jaén (Spain), and all subjects provided written informed consent. A total of 40 healthy students from the University of Jaén were previously selected for this study. Subjects who had one or more of the following conditions were excluded from the current study: contraindication to manipulation, history of whiplash or cervical surgery, pain related to cervical spine or arm in the previous month, subject under pharmacological treatment, subject who has practice strenuous exercise 7 days prior to the experiment, headache in the previous days, having undergone spinal manipulative therapy in the previous 2 months, or loss of standing balance. Information about eating and physical exercise habits was obtained from participants after the experiment was conducted.

2.3. Randomization and Intervention. A number was assigned to each participant by an external consultant, who did not have any further involvement in the research. After that, a list of random numbers ranging from 0 to 30 was generated by Microsoft Excel software. The first ten numbers of this list were assigned to the control group, the next ten numbers to the cervical manipulation group, and the last ten to the thoracic manipulation group. Randomization was done by an external assessor who did not participate in the research.

Thoracic SM technique involved a high-velocity, end-range, anterior-posterior force through the elbows to the middle thoracic spine (T3-T4) on the lower thoracic (T4-T5) spine in a supine position with patient's arms crossed. In the cervical manipulation, a high-velocity, midrange left rotational force to the midcervical spine (C4) on the lower (C5) cervical spine in supine, with left rotation and right side bending.

Control participants were treated following the cervical manipulation protocol with regard to hand contact, but without intention of mobilization, nor application of tissue tension by the treating clinician.

The time devoted to HVLA thrust manipulations in the intervention groups and that to the simulated procedures in control group were similar, in order to minimize the potential for an attention effect. All the researchers were blinded to the therapist's intervention.

2.4. Blood Extraction. Serum samples were extracted before intervention, right after intervention, and two hours after manipulation by venipuncture of the cephalic vein using a Vacutainer system (Becton-Dickinson, United Kingdom). Blood was collected in two different tubes for both serum (BD Vacutainer SST II Advance, ref. 367953) and plasma (BD Vacutainer LH PST II Advance, ref. 367374) separation. After blood extraction, tubes were let stand at room temperature for one hour until the blood clotted. Afterwards, tubes were centrifuged for ten minutes at $2000 \mathrm{~g}$ (Avanti J-30I, Beckman Coulter, USA). Supernatant was collected from the tubes. Three serum aliquots were done for the determination of CPK, LDH, CRP, aldolase, and NSE. Plasma was divided into two different aliquots for troponin-I and myoglobin assay. All the aliquots were kept at $-80^{\circ} \mathrm{C}$ until used.

2.5. Outcomes and Follow-Up. CPK, LDH, and aldolase serum concentrations were calculated by enzymatic assay in an OLYMPUS AU5400 Analyzer (Beckman-Coulter, USA). Troponin-I levels were measured through chemiluminescence (Dimension EXL, Siemens, Germany), as was neuron-specific enolase (LiasonAnalyzer, Dia-Sorin). CRP concentration was determined by turbidimetry assay (OLYMPUS AU5400 Analyzer, Beckman Coulter, USA). Myoglobin was determined by means of enzymatic immunoassay (Dimension EXL, Siemens, Germany). All the assays were carried out in the Ciudad de Jaén University Hospital following the manufacturer's protocol. 


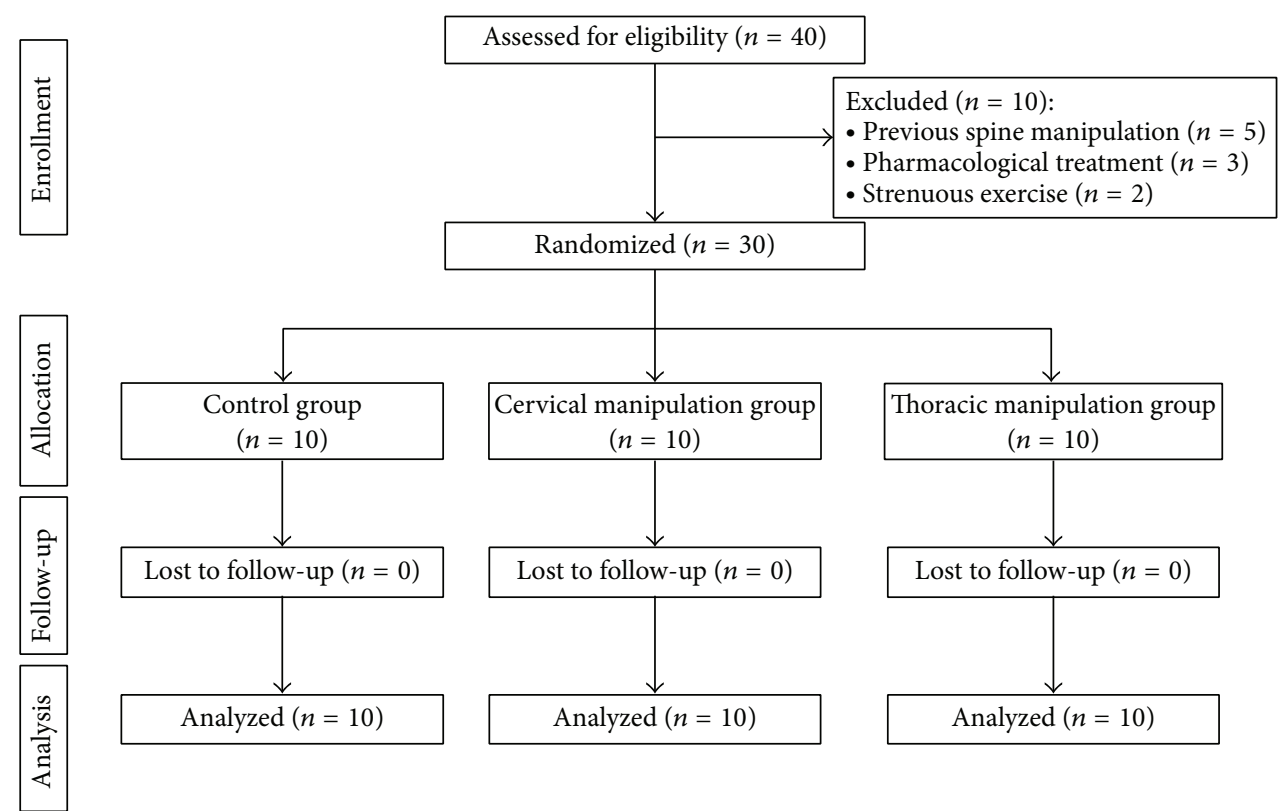

FIGURE 1: Flowchart diagram of the study.

2.6. Statistical Analysis. Continuous variables were described by means and standard deviation, and categorical variables by frequencies and percentages. Kolmogorov-Smirnov was used to verify the normal distribution of continuous variables in the groups, and Levene's test was used to confirm the homoscedasticity of the samples. One-way analysis of variance and Chi-squared test were used to prove comparability on sociodemographic baseline values. To prove the effect of the independent factor (control, thoracic, or cervical manipulation) on the dependent variables (blood concentrations) at each time point (pretreatment, zero hours posttreatment, and two hours posttreatment), a mixed-model ANOVA was employed. The hypothesis of interest was the group-by-time interaction. A separate $3 \times 3$ mixed-model ANOVA was applied for each dependent variable. In order to measure the effect size, eta-squared and Cohen's $d$ were used for groupby-time effect and pairwise comparisons, respectively. For Cohen's $d$ interpretation an effect size of 0.2 was considered small, 0.5 moderate, and 0.8 large [27]. Demographic and experimental data were treated with the software SPSS 19.0 (IBM, USA) and MedCalc12.7 (MedCalc, Belgium). All of the analyses were performed with a $95 \%$ confidence interval $(P<0.05)$.

\section{Results}

Of the 40 patients screened in the University of Jaén, a total of 30 subjects met the inclusion criteria and agreed to participate in the study. Ten participants were randomly assigned to each treatment group. Demographic data are displayed in Table 1. Figure 1 shows the flowchart depicting participant recruitment and retention. No differences were observed between groups at baseline measures. Only CPR shows a difference in the limits of significance. No vitamin supplementation was reported by the participants. They followed the Mediterranean diet [28] and had a sedentary lifestyle [29].

Descriptive data for all dependent variables in each group for each time point are shown in Table 2. Mixed-model ANOVA failed to reveal a group-by-time interaction in any of the dependent variables $(P>0.05)$. Effect sizes, measured with eta-squared, were small for all dependent variables and the interaction never explained more than $12 \%$ of the variance (Table 2). The higher effect was apparent for LDH (11.2\%) and the smaller was shown for aldolase (1.9\%).

The pairwise comparisons between control and both the thoracic and cervical manipulation groups show a result in the limits of statistical significance only for the comparison between control and thoracic groups in CRP at zero hours posttreatment and for myoglobin at two hours posttreatment (Table 3). However, these statistical significances have no real meaning because of the lack of statistical significance of the ANOVA. Nevertheless, the effect sizes could be considered high $(d>0.8)$ for the comparison between the control and the thoracic groups for CRP in all time points and for myoglobin at two hours posttreatment. In the comparison between control and cervical manipulation groups, only the effect size for myoglobin at two hours posttreatment could be considered high (Table 3 ). Troponin-I data are not shown because the value for the subjects was zero in all the timepoint and groups.

\section{Discussion}

Spine manipulation (SM) is a manual therapy technique commonly applied, which presents benefits for patients such as an anti-inflammatory effect [30], pain relief, and reduction of drug consumption [31]. However, some studies have proposed a noxious effect of SM application. In this sense, the reports 
TABLE 1: Sociodemographic characteristics and baseline measures of the groups.

\begin{tabular}{|c|c|c|c|c|c|c|c|c|}
\hline Characteristic & $\begin{array}{c}\text { Control } \\
n=10 \\
\end{array}$ & $\begin{array}{c}\text { Thoracic } \\
n=10 \\
\end{array}$ & $\begin{array}{c}\text { Cervical } \\
n=10\end{array}$ & & & & & $P$-value \\
\hline Age $^{*}$ (years) & $27.60 \pm 3.22$ & $29.80 \pm 3.52$ & $28.60 \pm 3.99$ & & & & & 0.195 \\
\hline $\mathrm{BMI}^{*}$ & $21.45 \pm 2.38$ & $23.98 \pm 3.97$ & $23.17 \pm 2.94$ & & & & & 0.206 \\
\hline Weight* (Kg) & $66.60 \pm 8.47$ & $73.70 \pm 14.33$ & $71.20 \pm 12.19$ & & & & & 0.528 \\
\hline Height* $^{*}(\mathrm{~m})$ & $1.72 \pm 0.07$ & $1.75 \pm 0.06$ & $1.75 \pm 0.12$ & & & & & 0.301 \\
\hline $\mathrm{CPK}^{*}(\mathrm{U} / \mathrm{L})$ & $74.90 \pm 17.21$ & $72.00 \pm 13.73$ & $65.10 \pm 14.78$ & & & & & 0.353 \\
\hline $\mathrm{LDH}^{*}(\mathrm{U} / \mathrm{L})$ & $276.03 \pm 57.30$ & $297.20 \pm 52.66$ & $275.90 \pm 43.40$ & & & & & 0.573 \\
\hline Enolase $^{*}(\mathrm{ng} / \mathrm{mL})$ & $9.48 \pm 1.81$ & $9.09 \pm 1.50$ & $9.57 \pm 3.26$ & & & & & 0.887 \\
\hline $\mathrm{CPR}^{*}(\mathrm{mg} / \mathrm{L})$ & $1.41 \pm 1.00$ & $0.56 \pm 0.30$ & $1.48 \pm 1.16$ & & & & & 0.056 \\
\hline Aldolase $^{*}(\mathrm{U} / \mathrm{L})$ & $3.09 \pm 1.60$ & $3.10 \pm 1.06$ & $3.13 \pm 1.02$ & & & & & 0.998 \\
\hline Myoglobin* (ng/mL) & $50.76 \pm 31.39$ & $36.80 \pm 10.81$ & $35.40 \pm 20.87$ & & & & & 0.262 \\
\hline \multirow{2}{*}{ Gender $^{\dagger}$} & Male & 6 & $60.0 \%$ & 5 & $50.0 \%$ & 5 & $50.0 \%$ & \multirow{2}{*}{0.875} \\
\hline & Female & 4 & $40.0 \%$ & 5 & $50.0 \%$ & 5 & $50.0 \%$ & \\
\hline
\end{tabular}

BMI: body mass index; CPK: creatine phosphokinase; LDH: lactate dehydrogenase; CRP: C-reactive protein.

${ }^{*}$ Continuous variables are expressed as a mean \pm standard deviation. $P$ values correspond to one-way ANOVA test.

${ }^{\dagger}$ Categorical variables are expressed as frequencies and percentages. $P$ values correspond to Chi-squared test.

TABLE 2: Blood concentrations for all groups at each follow-up period. Statistical significance and effect size for group-by-time interaction.

\begin{tabular}{|c|c|c|c|c|c|c|}
\hline & & $\begin{array}{c}\text { Pre-T } \\
\text { Mean } \pm \text { SD }\end{array}$ & $\begin{array}{c}\text { Post-0 H } \\
\text { Mean } \pm \text { SD }\end{array}$ & $\begin{array}{c}\text { Post } 2 \mathrm{H} \\
\text { Mean } \pm \text { SD }\end{array}$ & $P$ value & $\mathrm{Eta}^{2}$ \\
\hline \multirow{3}{*}{ CPK (U/L) } & Control & $74.90 \pm 17.21$ & $74.10 \pm 17.12$ & $74.40 \pm 16.57$ & \multirow{3}{*}{0.425} & \multirow{3}{*}{0.065} \\
\hline & Thoracic & $72.00 \pm 13.73$ & $72.10 \pm 16.00$ & $71.10 \pm 19.09$ & & \\
\hline & Cervical & $65.10 \pm 14.78$ & $65.20 \pm 16.29$ & $68.10 \pm 16.38$ & & \\
\hline \multirow{3}{*}{ LDH (U/L) } & Control & $276.03 \pm 57.30$ & $283.43 \pm 44.29$ & $268.11 \pm 47.02$ & \multirow{3}{*}{0.167} & \multirow{3}{*}{0.112} \\
\hline & Thoracic & $297.20 \pm 52.66$ & $294.70 \pm 52.69$ & $289.80 \pm 67.36$ & & \\
\hline & Cervical & $275.90 \pm 43.40$ & $276.00 \pm 28.20$ & $302.80 \pm 82.64$ & & \\
\hline \multirow{3}{*}{ Enolase (ng/mL) } & Control & $9.48 \pm 1.81$ & $8.90 \pm 1.24$ & $8.86 \pm 1.29$ & \multirow{3}{*}{0.235} & \multirow{3}{*}{0.100} \\
\hline & Thoracic & $9.09 \pm 1.50$ & $9.52 \pm 1.41$ & $9.42 \pm 2.95$ & & \\
\hline & Cervical & $9.57 \pm 3.26$ & $9.23 \pm 1.97$ & $11.57 \pm 6.17$ & & \\
\hline \multirow{3}{*}{ CPR (mg/L) } & Control & $1.41 \pm 1.00$ & $1.61 \pm 1.02$ & $1.53 \pm 1.19$ & \multirow{3}{*}{0.486} & \multirow{3}{*}{0.058} \\
\hline & Thoracic & $0.56 \pm 0.30$ & $0.50 \pm 0.27$ & $0.50 \pm 0.30$ & & \\
\hline & Cervical & $1.48 \pm 1.16$ & $1.48 \pm 1.22$ & $1.47 \pm 1.15$ & & \\
\hline \multirow{3}{*}{ Aldolase (U/L) } & Control & $3.09 \pm 1.60$ & $3.36 \pm 1.37$ & $3.50 \pm 1.57$ & \multirow{3}{*}{0.859} & \multirow{3}{*}{0.019} \\
\hline & Thoracic & $3.10 \pm 1.06$ & $3.23 \pm 1.25$ & $3.17 \pm 1.37$ & & \\
\hline & Cervical & $3.13 \pm 1.02$ & $3.29 \pm 0.90$ & $3.63 \pm 1.38$ & & \\
\hline \multirow{3}{*}{ Myoglobin (ng/mL) } & Control & $50.76 \pm 31.39$ & $52.18 \pm 23.63$ & $70.20 \pm 43.56$ & \multirow{3}{*}{0.312} & \multirow{3}{*}{0.083} \\
\hline & Thoracic & $36.80 \pm 10.81$ & $38.30 \pm 10.80$ & $36.60 \pm 10.60$ & & \\
\hline & Cervical & $35.40 \pm 20.87$ & $35.80 \pm 19.62$ & $39.10 \pm 22.19$ & & \\
\hline
\end{tabular}

Pre-T: pretreatment values; Post- $0 \mathrm{H}$ : values $0 \mathrm{H}$ after intervention; Post- $2 \mathrm{H}$ : values $2 \mathrm{H}$ after intervention; CPK: creatine phosphokinase; LDH: lactate dehydrogenase; CRP: C-reactive protein.

on its effects on inducing tissue injuries are controversial, as it has been related to adverse events $[7,12,32,33]$. The literature points at catastrophic manual-therapy-induced adverse events being dependent on tissue damage. In this sense, the detection of some proteins in blood samples has been revealed as useful in some musculoskeletal and neurological conditions [34-36] to detect tissue damage. Thus, the present work is focused on the determination of biological damage markers in blood samples after a cervical or a thoracic manipulation, in order to corroborate whether or not spinal manipulation causes measurable tissue damage.

Adverse events from manual therapy range from the catastrophic, such as cervical artery dissection producing a stroke, through bruising to muscle soreness that could be regarded as a minor, and expected, consequence of treatment [37]. Rubinstein et al. reported that $72 \%$ of adverse events occurred after the first treatment [38]. Most adverse events reported by manual therapy patients are thought to be benign and 
TABLE 3: Mean differences between control and both the thoracic and the cervical groups.

\begin{tabular}{|c|c|c|c|c|c|c|c|c|c|}
\hline & & \multicolumn{4}{|c|}{ Control-thoracic } & \multicolumn{4}{|c|}{ Control-cervical } \\
\hline & & Mean difference & $95 \% \mathrm{CI}$ & $P$ value & Cohen- $D$ & $\begin{array}{c}\text { Mean } \\
\text { difference }\end{array}$ & $95 \%$ CI & $P$ value & Cohen- $D$ \\
\hline \multirow{3}{*}{ CPK (U/L) } & Pretreatment & 2.90 & $(-14.57 ; 20.37)$ & 1.000 & 0.19 & 9.80 & $(-7.67 ; 27.27)$ & 0.491 & 0.61 \\
\hline & Post $0 \mathrm{H}$ & 2.00 & $(-16.81 ; 20.81)$ & 1.000 & 0.12 & 8.90 & $(-9.91 ; 27.71)$ & 0.713 & 0.53 \\
\hline & Post $2 \mathrm{H}$ & 3.30 & $(-16.55 ; 23.15)$ & 1.000 & 0.18 & 6.30 & $(-13.55 ; 26.15)$ & 1.000 & 0.38 \\
\hline \multirow{3}{*}{$\mathrm{LDH}(\mathrm{U} / \mathrm{L})$} & Pretreatment & -21.17 & $(-79.90 ; 37.55)$ & 1.000 & 0.38 & 0.13 & $(-58.60 ; 58.85)$ & 1.000 & 0.00 \\
\hline & Post $0 \mathrm{H}$ & -11.27 & $(-60.29 ; 37.75)$ & 1.000 & 0.23 & 7.43 & $(-41.59 ; 56.45)$ & 1.000 & 0.20 \\
\hline & Post $2 \mathrm{H}$ & -21.69 & $(-98.48 ; 55.11)$ & 1.000 & 0.37 & -34.69 & $(-111.48 ; 42.11)$ & 0.777 & 0.52 \\
\hline \multirow{3}{*}{ Enolase (ng/mL) } & Pretreatment & 0.39 & $(-2.26 ; 3.04)$ & 1.000 & 0.23 & -0.09 & $(-2.74 ; 2.56)$ & 1.000 & 0.04 \\
\hline & Post $0 \mathrm{H}$ & -0.62 & $(-2.42 ; 1.17)$ & 1.000 & 0.47 & -0.33 & $(-2.13 ; 1.46)$ & 1.000 & 0.20 \\
\hline & Post $2 \mathrm{H}$ & -0.56 & $(-5.15 ; 4.03)$ & 1.000 & 0.24 & -2.71 & $(-7.30 ; 1.88)$ & 0.431 & 0.61 \\
\hline \multirow{3}{*}{ CRP (mg/L) } & Pretreatment & 0.85 & $(-0.18 ; 1.88)$ & 0.136 & 1.14 & -0.07 & $(-1.10 ; 0.96)$ & 1.000 & 0.07 \\
\hline & Post $0 \mathrm{H}$ & 1.11 & $(0.05 ; 2.18)$ & 0.039 & 1.49 & 0.13 & $(-0.93 ; 1.20)$ & 1.000 & 0.12 \\
\hline & Post $2 \mathrm{H}$ & 1.03 & $(-0.09 ; 2.14)$ & 0.078 & 1.18 & 0.06 & $(-1.06 ; 1.17)$ & 1.000 & 0.05 \\
\hline \multirow{3}{*}{ Aldolase (U/L) } & Pretreatment & -0.01 & $(-1.44 ; 1.43)$ & 1.000 & 0.00 & -0.04 & $(-1.47 ; 1.40)$ & 1.000 & 0.03 \\
\hline & Post $0 \mathrm{H}$ & 0.13 & $(-1.23 ; 1.49)$ & 1.000 & 0.10 & 0.07 & $(-1.29 ; 1.43)$ & 1.000 & 0.06 \\
\hline & Post $2 \mathrm{H}$ & 0.33 & $(-1.32 ; 1.98)$ & 1.000 & 0.22 & -0.13 & $(-1.78 ; 1.52)$ & 1.000 & 0.09 \\
\hline \multirow{3}{*}{ Myoglobin (ng/mL) } & Pretreatment & 13.96 & $(-11.89 ; 39.80)$ & 0.538 & 0.59 & 15.36 & $(-10.49 ; 41.20)$ & 0.423 & 0.58 \\
\hline & Post $0 \mathrm{H}$ & 13.88 & $(-7.57 ; 35.34)$ & 0.331 & 0.76 & 16.38 & $(-5.07 ; 37.84)$ & 0.185 & 0.75 \\
\hline & Post $2 \mathrm{H}$ & 33.60 & $(0.63 ; 66.57)$ & $0.045^{*}$ & 1.06 & 31.10 & $(-1.87 ; 64.07)$ & 0.069 & 0.90 \\
\hline
\end{tabular}

Pre-T: pretreatment values; Post- $0 \mathrm{H}$ : values $0 \mathrm{H}$ after intervention; Post- $2 \mathrm{H}$ : values $2 \mathrm{H}$ after intervention; CPK: creatine phosphokinase; LDH: lactate dehydrogenase; CRP: C-reactive protein.

${ }^{*}$ Trend to statistical significance.

transient and are often unknown to the practitioner unless patients show observable signs (e.g., loss of motion or neurological deficits) or report pain or discomfort [39]. A recent systematic review shows that nearly half of patients experience adverse events after manual therapy. These adverse events are short-lived and minor, and most will occur within 24 hours and resolve within 72 hours. The relative risk of these adverse events is similar for manual therapy plus exercise treatment and for sham/passive/control interventions [37].

The biomarkers used in the study are generally used to detect tissue damage. Creatine phosphokinase (CPK) is an intracellular enzyme related to energy metabolism, and its level in serum has been extensively used as a diagnostic marker for muscle injury [18]. Myoglobin and troponin-I are sensitive markers for skeletal muscle or cardiac muscle damage [19]. Both aldolase and lactate dehydrogenase (LDH) are biomarkers for general tissue damage. The presence of neuron-specific enolase (NSE) in plasma samples is used as a diffuse neuronal damage marker [40]. The C-reactive protein (CRP) is a nonspecific marker of inflammation. In fact, Huang et al. showed that elevated mechanical strain on vessels could induce the local expression of proinflammatory cytokines like CRP [41]. These parameters had been extensively used in research in order to analyze the effect of active physical therapy in the expression of tissue damage markers $[42,43]$. After the analysis of seven tissue damage markers, our data do not show any significant differences in CPK, LDH, troponin-I, myoglobin, aldolase, NSE, and CRP concentrations. A mixed-model ANOVA failed to reveal a group-by-time interaction in any of the dependent variables $(P>0.05)$. The pairwise comparisons between the control and both the thoracic and the cervical manipulation groups show lack of statistical significance except for two comparisons in the limit of statistical significance (which lack real meaning). The relatively high effect size found in the comparison of CRP levels between the control group and the thoracic manipulation group was already apparent at pretreatment and did not increase significantly in the two posttreatment measurements. The behavior of these parameters suggests that the mechanical stimulus induced by spinal manipulation alone is not enough to provoke cell damage or tissue breakage in healthy subjects. These data agree with other works that show no alteration in pathologic blood vessels after a cervical manipulation [44]. Nevertheless, Huang et al. showed that mechanical strain increased CRP expression in the saphenous vein in a strength-dependent manner [41]. Rather than an episode of mechanical stretch, some studies propose that a repetitive mechanical stress was needed to induce microstructural damage in vessels [45]. Moreover, studies demonstrated that the mechanical load of the vertebral artery during SM application was almost an order of magnitude lower than the strain required to cause its mechanical disruption [14].

The findings of the present study contradict those of previous studies on other manual therapies modalities. Danneskiold-Samsøe et al. identified an increase on serum myoglobin 
after a deep massage [46, 47]. Arkko et al. also found increases of serum CPK and LDH concentrations [48]. This contradiction may be due to differences between modalities or because the studies of Danneskiold-Samsøe and Arkko were conducted on nonhealthy subjects whereas our subjects were healthy. Moreover, the deep massage protocol used in these studies differs from our manipulation technique in the application time of the treatment protocol. The process for a single spinal manipulation takes a few seconds but, in contrast, massage protocol takes some minutes. It is therefore possible that the long time during which mechanical force was directly applied to tissues could explain the observed increase in myoglobin, CPK, and LDH in these studies $[4,38,39]$.

After the manipulation, two of the subjects suffered syncope. Both belonged to the thoracic manipulative group. It is likely that syncope after spinal manipulative therapy is not related to tissue damage and that such adverse event may be explained by other reasons.

Strengths and Limitations of the Study. First, the sample was small due to methodological issues, making it difficult to generalize our results. Second, it was not possible to blind the clinician or the subjects due to the nature of the intervention, which constitutes a risk of bias. Third, the present study was conducted on asymptomatic subjects, so it is not possible to extrapolate the present findings to a symptomatic population.

\section{Conclusions}

Our data show no changes in any of the studied damage markers. Although this study examined the outcomes in an asymptomatic population, lower cervical and thoracic manipulative techniques seem to be safe manual therapies techniques which cause no harm to the health of the subject. These data may be used as evidence of the safe application of spinal manipulation to healthy subjects. Further studies with a large sample size and a patient population are needed to corroborate the innocuous effects of spinal manipulation.

\section{Conflict of Interests}

The authors declare that there is no conflict of interests regarding the publication of this paper.

\section{Acknowledgment}

The authors wish to thank all the professionals of the Clinical Analysis Service of the Hospital Ciudad de Jaén (Jaén, Spain) for collaborating in this study.

\section{References}

[1] D. W. Evans and N. Lucas, "What is "manipulation"? A reappraisal," Manual Therapy, vol. 15, no. 3, pp. 286-291, 2010.

[2] M. I. Gatterman and D. T. Hansen, "Development of chiropractic nomenclature through consensus," Journal of Manipulative and Physiological Therapeutics, vol. 17, no. 5, pp. 302-309, 1994.

[3] J. J. Kuczynski, B. Schwieterman, K. Columber, D. Knupp, L. Shaub, and C. E. Cook, "Effectiveness of physical therapist administered spinal manipulation for the treatment of low back pain: a systematic review of the literature," International Journal of Sports Physical Therapy, vol. 7, no. 6, pp. 647-662, 2012.

[4] R. Martínez-Segura, C. Fernández-de-las-Peñas, M. RuizSáez, C. López-Jiménez, and C. Rodríguez-Blanco, "Immediate effects on neck pain and active range of motion after a single cervical high-velocity low-amplitude manipulation in subjects presenting with mechanical neck pain: a randomized controlled trial," Journal of Manipulative and Physiological Therapeutics, vol. 29, no. 7, pp. 511-517, 2006.

[5] S. Haldeman, F. J. Kohlbeck, and M. McGregor, "Risk factors and precipitating neck movements causing vertebrobasilar artery dissection after cervical trauma and spinal manipulation," Spine, vol. 24, no. 8, pp. 785-794, 1999.

[6] C. Stevinson, W. Honan, B. Cooke, and E. Ernst, "Neurological complications of cervical spine manipulation," Journal of the Royal Society of Medicine, vol. 94, no. 3, pp. 107-110, 2001.

[7] E. Ernst, "Adverse effects of spinal manipulation: a systematic review," Journal of the Royal Society of Medicine, vol. 100, no. 7, pp. 330-338, 2007.

[8] M. Paciaroni and J. Bogousslavsky, "Cerebrovascular complications of neck manipulation,” European Neurology, vol. 61, no. 2, pp. 112-118, 2009.

[9] S. Wuest, B. Symons, T. Leonard, and W. Herzog, "Preliminary report: biomechanics of vertebral artery segments C1-C6 during cervical spinal manipulation," Journal of Manipulative and Physiological Therapeutics, vol. 33, no. 4, pp. 273-278, 2010.

[10] B. Cagnie, E. Barbaix, E. Vinck, K. D'Herde, and D. Cambier, "Atherosclerosis in the vertebral artery: an intrinsic risk factor in the use of spinal manipulation?" Surgical and Radiologic Anatomy, vol. 28, no. 2, pp. 129-134, 2006.

[11] B. Symons and W. Herzog, "Cervical artery dissection: a biomechanical perspective," The Journal of the Canadian Chiropractic Association, vol. 57, no. 4, pp. 276-278, 2013.

[12] E. Ernst, "Vascular accidents after neck manipulation: cause or coincidence?" International Journal of Clinical Practice, vol. 64, no. 6, pp. 673-677, 2010.

[13] S. Haldeman, F. J. Kohlbeck, and M. McGregor, "Unpredictability of cerebrovascular ischemia associated with cervical spine manipulation therapy: a review of sixty-four cases after cervical spine manipulation," Spine, vol. 27, no. 1, pp. 49-55, 2002.

[14] B. P. Symons, T. Leonard, and W. Herzog, "Internal forces sustained by the vertebral artery during spinal manipulative therapy," Journal of Manipulative and Physiological Therapeutics, vol. 25, no. 8, pp. 504-510, 2002.

[15] C. Fernández-de-las-Peñas, C. Alonso-Blanco, J. A. Cleland, C. Rodríguez-Blanco, and F. Alburquerque-Sendín, "Changes in pressure pain thresholds over C5-C6 zygapophyseal joint after a cervicothoracic junction manipulation in healthy subjects," Journal of Manipulative and Physiological Therapeutics, vol. 31, no. 5, pp. 332-337, 2008.

[16] P. Brancaccio, G. Lippi, and N. Maffulli, "Biochemical markers of muscular damage," Clinical Chemistry and Laboratory Medicine, vol. 48, no. 6, pp. 757-767, 2010.

[17] M. Kaundal and S. Sharma, "Analysis of isoproterenol-induced changes in gastrocnemius muscle and serum lactate dehydrogenase expression in mice," Singapore Medical Journal, vol. 52, no. 4, pp. 274-282, 2011.

[18] J. Nie, T. K. Tong, K. George, F. H. Fu, H. Lin, and Q. Shi, "Resting and post-exercise serum biomarkers of cardiac and skeletal muscle damage in adolescent runners," Scandinavian 
Journal of Medicine and Science in Sports, vol. 21, no. 5, pp. 625629, 2011.

[19] F. A. Van Nieuwenhoven, A. H. Kleine, K. W. H. Wodzig et al., "Discrimination between myocardial and skeletal muscle injury by assessment of the plasma ratio of myoglobin over fatty acidbinding protein," Circulation, vol. 92, no. 10, pp. 2848-2854, 1995.

[20] E. S. D. Dos Santos, M. P. Pereira, L. Minuzzo et al., "Electrical cardioversion and myocardial injury: evaluation by new cardiac injury markers," Arquivos Brasileiros de Cardiologia, vol. 86, no. 3, pp. 191-197, 2006.

[21] E. G. Zandbergen, R. J. de Haan, and A. Hijdra, "Systematic review of prediction of poor outcome in anoxic-ischaemic coma with biochemical markers of brain damage," Intensive Care Medicine, vol. 27, no. 10, pp. 1661-1667, 2001.

[22] M. F. Baird, S. M. Graham, J. S. Baker, and G. F. Bickerstaff, "Creatine-kinase- and exercise-related muscle damage implications for muscle performance and recovery," Journal of Nutrition and Metabolism, vol. 2012, Article ID 960363, 13 pages, 2012.

[23] S. Sorichter, J. Mair, A. Koller et al., "Skeletal troponin I as a marker of exercise-induced muscle damage," Journal of Applied Physiology, vol. 83, no. 4, pp. 1076-1082, 1997.

[24] S. Sorichter, J. Mair, A. Koller et al., "Release of muscle proteins after downhill running in male and female subjects," Scandinavian Journal of Medicine and Science in Sports, vol. 11, no. 1, pp. 28-32, 2001.

[25] P. Gradisek, J. Osredkar, M. Korsic, and B. Kremzar, "Multiple indicators model of long-term mortality in traumatic brain injury," Brain Injury, vol. 26, no. 12, pp. 1472-1481, 2012.

[26] M. B. Pepys and G. M. Hirschfield, "C-reactive protein: a critical update," The Journal of Clinical Investigation, vol. 111, no. 12, pp. 1805-1812, 2003.

[27] J. Cohen, Statistical Power Analysis for the Behavioural Sciences, Lawrence Erlbaum Associates, Hillsdale, NJ, USA, 1988.

[28] L. Serra-Majem, A. Trichopoulou, J. N. De La Cruz et al., "International Task Force on the Mediterranean Diet. Does the definition of the Mediterranean diet need to be updated?" Public Health Nutrition, vol. 7, no. 7, pp. 927-929, 2004.

[29] R. R. Pate, J. R. O'Neill, and F. Lobelo, “The evolving definition of "sedentary"', Exercise and Sport Sciences Reviews, vol. 36, no. 4, pp. 173-178, 2008.

[30] R. A. Roy, J. P. Boucher, and A. S. Comtois, "Inflammatory response following a short-term course of chiropractic treatment in subjects with and without chronic low back pain," Journal of Chiropractic Medicine, vol. 9, no. 3, pp. 107-114, 2010.

[31] L. G. F. Giles and R. Muller, "Chronic spinal pain: a randomized clinical trial comparing medication, acupuncture, and spinal manipulation," Spine, vol. 28, no. 14, pp. 1490-1503, 2003.

[32] F. C. Albuquerque, Y. C. Hu, S. R. Dashti et al., "Craniocervical arterial dissections as sequelae of chiropractic manipulation: patterns of injury and management," Journal of Neurosurgery, vol. 115, no. 6, pp. 1197-1205, 2011.

[33] J. J. Hebert, N. J. Stomski, S. D. French, and S. M. Rubinstein, "Serious adverse events and spinal manipulative therapy of the low back region: a systematic review of cases," Journal of Manipulative and Physiological Therapeutics, 2013.

[34] K. L. Flann, P. C. Lastayo, D. A. McClain, M. Hazel, and S. L. Lindstedt, "Muscle damage and muscle remodeling: no pain, no gain?" Journal of Experimental Biology, vol. 214, no. 4, pp. 674679, 2011.
[35] M. Herrmann, N. Curio, S. Jost et al., "Release of biochemical markers of damage to neuronal and glial brain tissue is associated with short and long term neuropsychological outcome after traumatic brain injury," Journal of Neurology Neurosurgery and Psychiatry, vol. 70, no. 1, pp. 95-100, 2001.

[36] A. J. McKune, S. J. Semple, and E. M. Peters-Futre, "Acute exercise-induced muscle injury," Biology of Sport, vol. 29, no. 1, pp. 3-10, 2012.

[37] D. Carnes, T. S. Mars, B. Mullinger, R. Froud, and M. Underwood, "Adverse events and manual therapy: a systematic review," Manual Therapy, vol. 15, no. 4, pp. 355-363, 2010.

[38] S. M. Rubinstein, C. Leboeuf-Yde, D. L. Knol, T. E. de Koekkoek, C. E. Pfeifle, and M. W. van Tulder, "The benefits outweigh the risks for patients undergoing chiropractic care for neck pain: a prospective, multicenter, cohort study," Journal of Manipulative and Physiological Therapeutics, vol. 30, no. 6, pp. 408-418, 2007.

[39] L. C. Carlesso, J. Cairney, L. Dolovich, and J. Hoogenes, "Defining adverse events in manual therapy: an exploratory qualitative analysis of the patient perspective," Manual Therapy, vol. 16, no. 5, pp. 440-446, 2011.

[40] S.-Y. Lee, Y.-C. Choi, J.-H. Kim, and W.-J. Kim, "Serum neuronspecific enolase level as a biomarker in differential diagnosis of seizure and syncope," Journal of Neurology, vol. 257, no. 10, pp. 1708-1712, 2010.

[41] G. Huang, C. Luo, X. Gu et al., "Mechanical strain induces expression of C-reactive protein in human blood vessels," Journal of Pharmacology and Experimental Therapeutics, vol. 330, no. 1, pp. 206-211, 2009.

[42] H. Alexanderson, C. H. Stenström, G. Jenner, and I. Lundberg, "The safety of a resistive home exercise program in patients with recent onset active polymyositis or dermatomyositis," Scandinavian Journal of Rheumatology, vol. 29, no. 5, pp. 295301, 2000.

[43] H. Alexanderson, L. A. Munters, M. Dastmalchi et al., "Resistive home exercise in patients with recent-onset polymyositis and dermatomyositis - a randomized controlled single-blinded study with a 2-year followup," Journal of Rheumatology, vol. 41, no. 6, pp. 1124-1132, 2014.

[44] S. Wynd, T. Anderson, and G. Kawchuk, "Effect of cervical spine manipulation on a pre-existing vascular lesion within the canine vertebral artery," Cerebrovascular Diseases, vol. 26, no. 3, pp. 304-309, 2008.

[45] N. Austin, L. M. DiFrancesco, and W. Herzog, "Microstructural damage in arterial tissue exposed to repeated tensile strains," Journal of Manipulative and Physiological Therapeutics, vol. 33, no. 1, pp. 14-19, 2010.

[46] B. Danneskiold-Samsøe, E. Christiansen, B. Lund, and R. B. Andersen, "Regional muscle tension and pain ("fibrositis"). Effect of massage on myoglobin in plasma," Scandinavian Journal of Rehabilitation Medicine, vol. 15, no. 1, pp. 17-20, 1983.

[47] B. Danneskiold-Samsøe, E. Christiansen, and R. Bach Andersen, "Myofascial pain and the role of myoglobin," Scandinavian Journal of Rheumatology, vol. 15, no. 2, pp. 174-178, 1986.

[48] P. J. Arkko, A. J. Pakarinen, and O. Kari Koskinen, "Effects of whole body massage on serum protein, electrolyte and hormone concentrations, enzyme activities, and hematological parameters," International Journal of Sports Medicine, vol. 4, no. 4, pp. 265-267, 1983. 


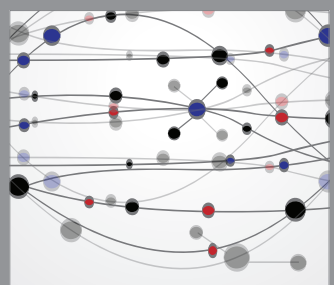

The Scientific World Journal
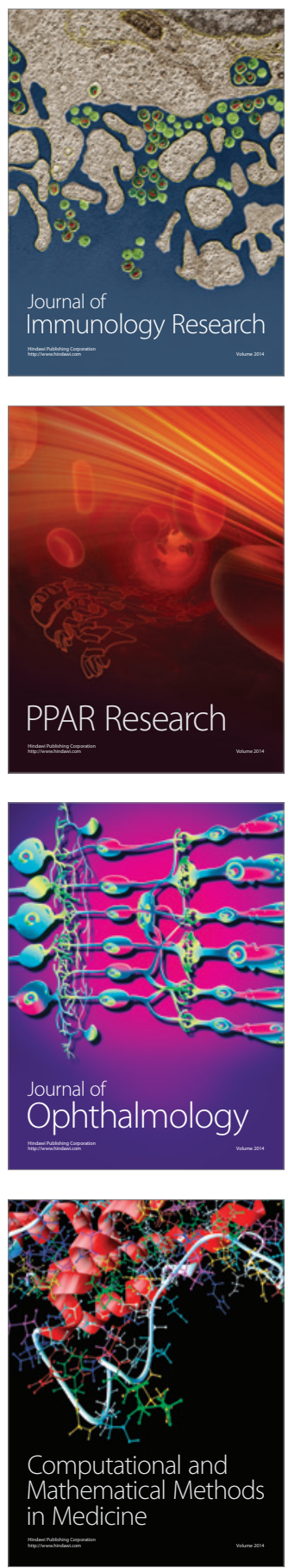

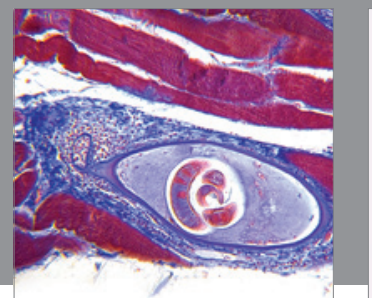

Gastroenterology

Research and Practice
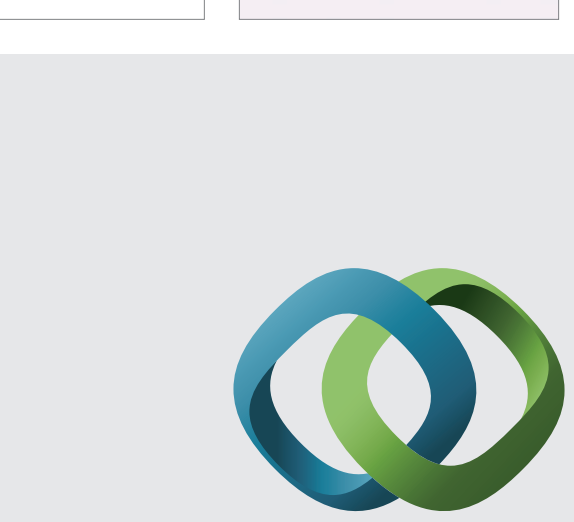

\section{Hindawi}

Submit your manuscripts at

http://www.hindawi.com
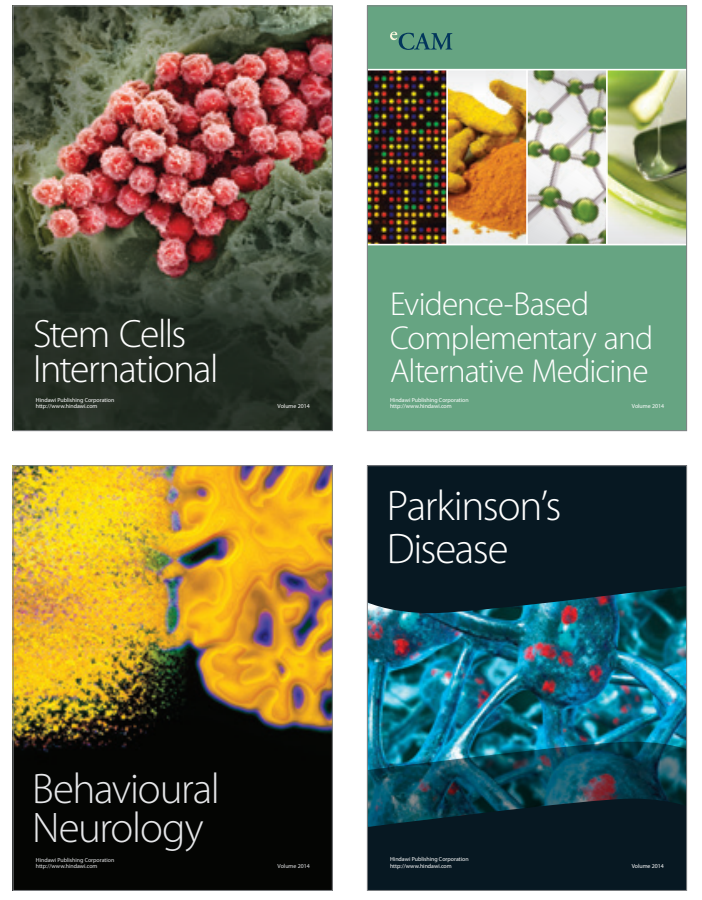
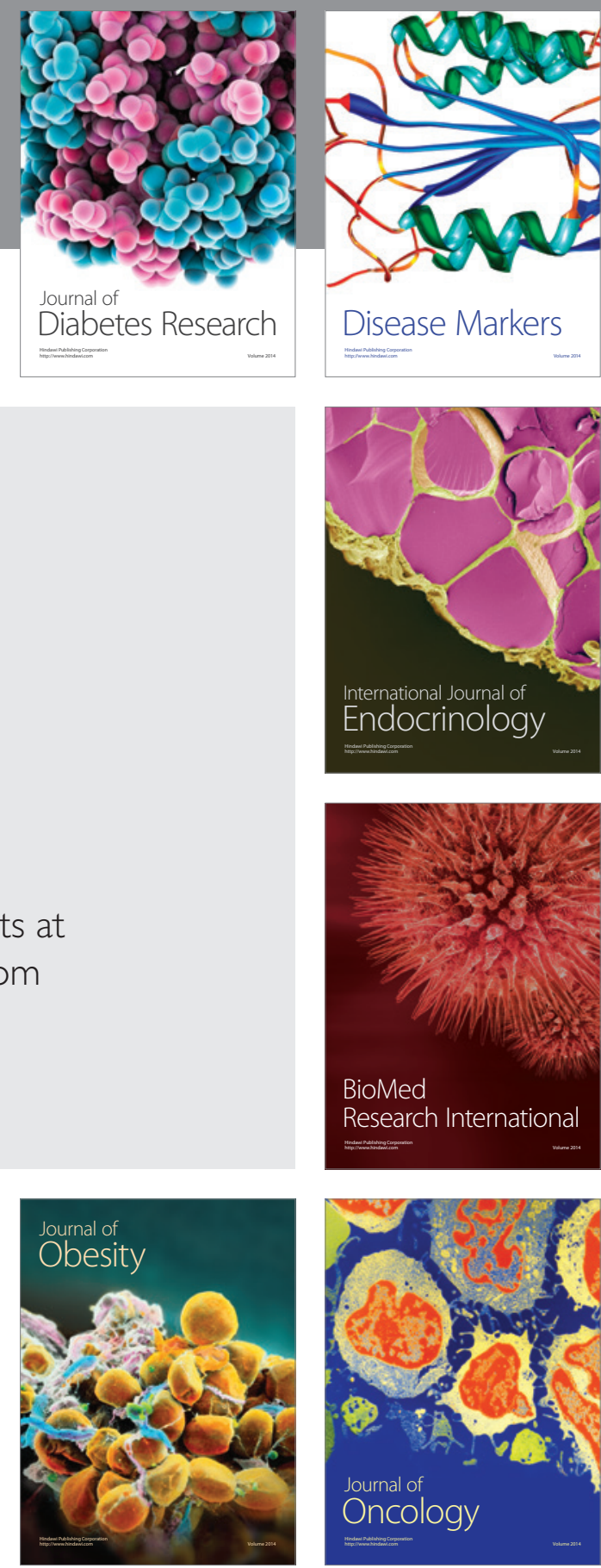

Disease Markers
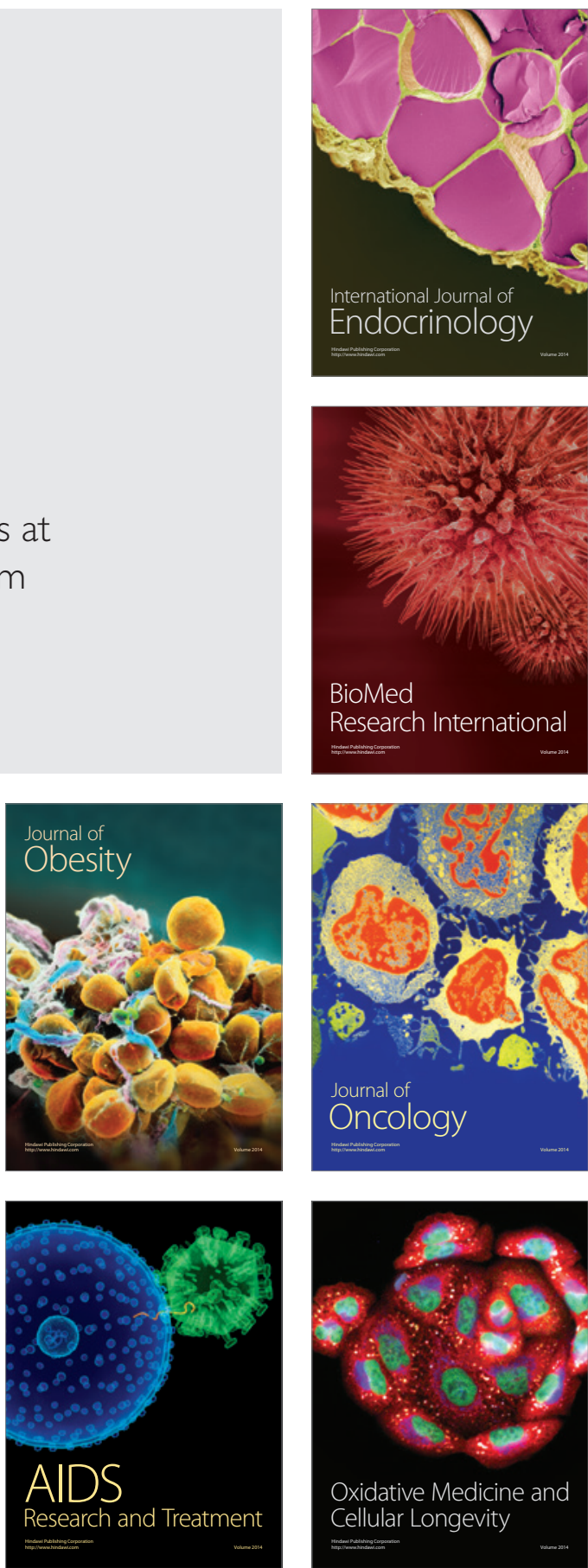